\title{
Magnetic field evolves to gravity field Part 2: particles
}

\author{
John C. Hodge $\mathrm{e}^{1 *}$ \\ ${ }^{1}$ Retired, 477 Mincey Rd., Franklin, NC, 28734
}

August 21, 2019

\begin{abstract}
The characterization of magnetic fields around elementary particles is an extension of a project to model the gravitational field. The observation that the South Pole is slightly stronger than the North Pole as suggested in a previous experiment is confirmed. Each of the particles has a unique magnetic field lobes structure.
\end{abstract}

keywords: magnetism, gravity, particles

\section{INTRODUCTION}

The Scalar Theory of Everything (STOE) posits the magnetic field of particles evolves into the gravitational field at macro scales. Particles were postulated to be assemblies of hods that are magnets. Accordingly, models of photons, neutrons, electrons, and positrons were suggested (Hodge 2016a).

This Paper continues the study that began to characterize the magnetic field in Hodge (2019). The experiments are in section 2. The Discussion and Conclusion are in section 3 .

\section{THE EXPERIMENT}

The same experiment setup that was used in Hodge (2019) is used in these experiments. Four disk magnets on the lower support is the probe of the magnetic field strength. The South Pole of the bottom magnet was oriented upward. The probe (bottom magnet) had 4 disks which is too strong for the fixture to have accurate measurements of Zone $1\left(d^{-1}\right)$.

*E-mail: jchodge@frontier.com 


\subsection{EXPERIMENT 1: PHOTON}

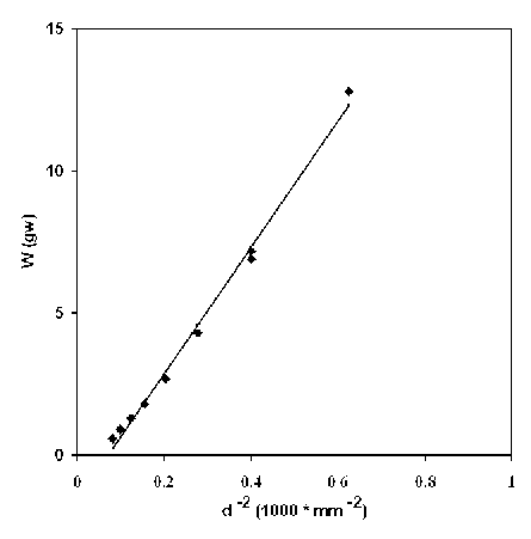

Figure 1: Plot of the $W$ versus $d^{-2}$ for 4 disks in the lower support and 6 disks in the upper support.

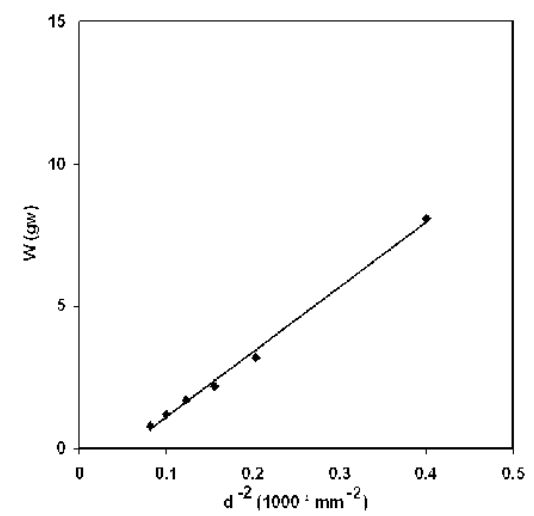

Figure 3: Plot of the $W$ versus $d^{-2}$ for 4 disks in the lower support and 8 disks in the upper support.

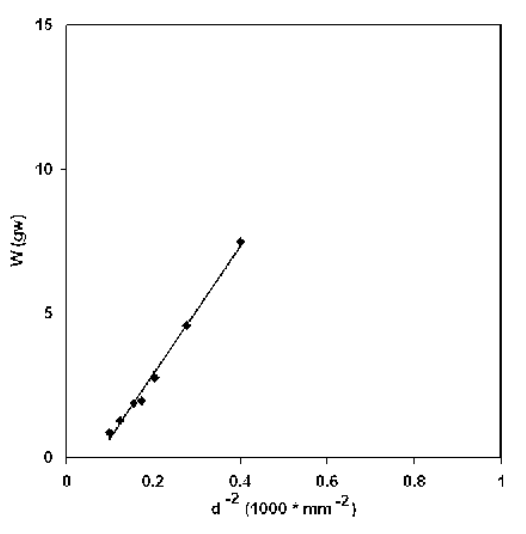

Figure 2: Plot of the $W$ versus $d^{-2}$ for 4 disks in the lower support and 7 disks in the upper support.

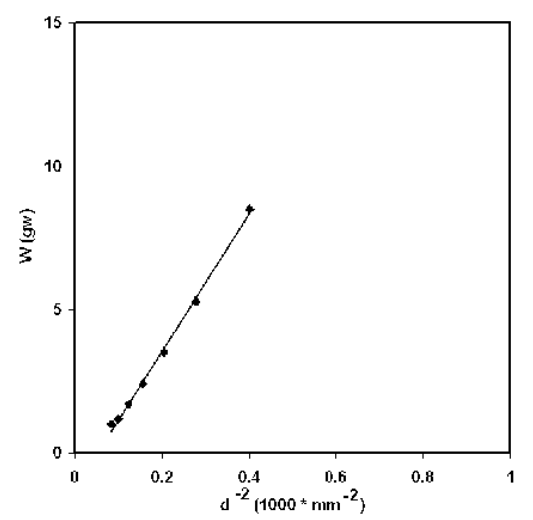

Figure 4: Plot of the $W$ versus $d^{-2}$ for 4 disks in the lower support and 10 disks in the upper support. 


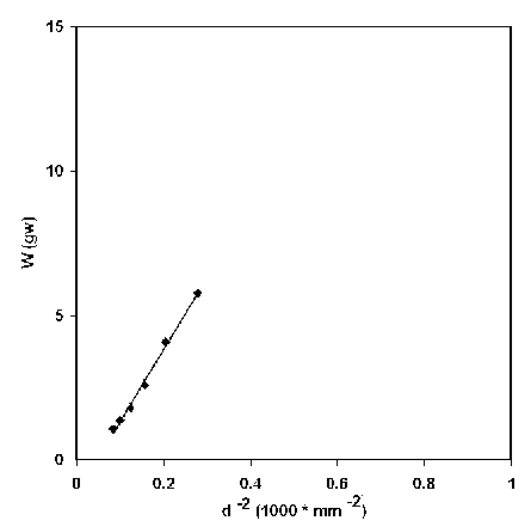

Figure 5: Plot of the $W$ versus $d^{-2}$ for 4 disks in the lower support and 11 disks in the upper support.

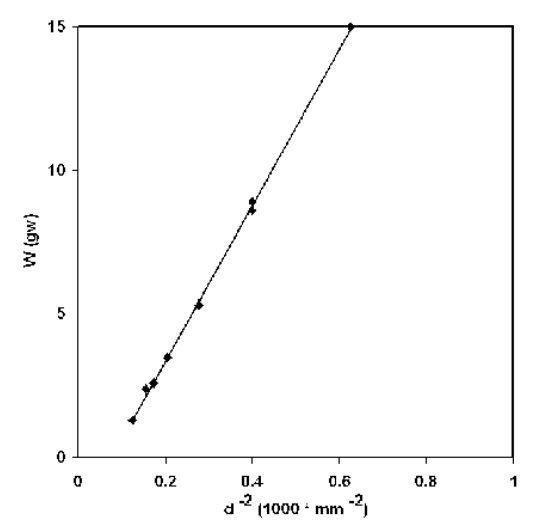

Figure 7: Plot of the $W$ versus $d^{-2}$ for 4 disks in the lower support and 15 disks in the upper support.

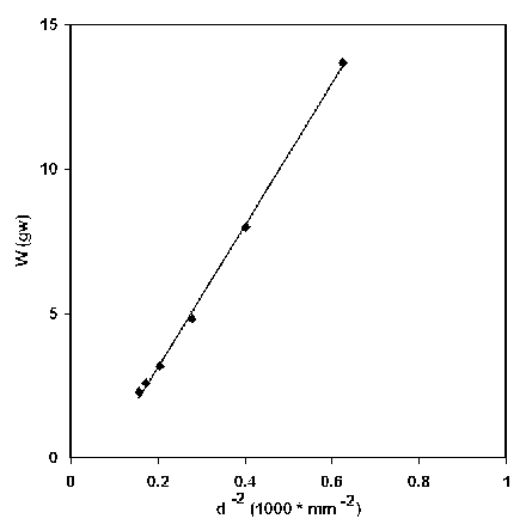

Figure 6: Plot of the $W$ versus $d^{-2}$ for 4 disks in the lower support and 12 disks in the upper support.

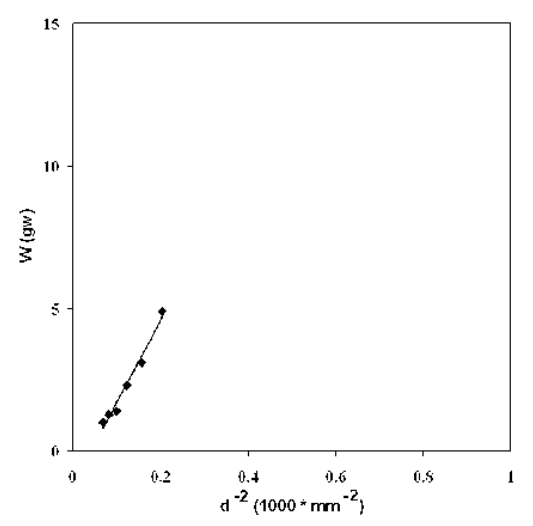

Figure 8: Plot of the $W$ versus $d^{-2}$ for 4 disks in the lower support and 20 disks in the upper support. 


\section{THE EXPERIMENT}

The equations for the best-fit lines at $1 \sigma$ confidence are:

Fig. $1 W(4 t o 6)=\left[22.2\left(1000 / d^{2}\right)-1.6\right] \pm 0.1 \mathrm{gw}$

Fig. $2 W(4 t o 7)=\left[22.3\left(1000 / d^{2}\right)-1.6\right] \pm 0.1 \mathrm{gw}$

Fig. $3 W(4 t o 8)=\left[23.0\left(1000 / d^{2}\right)-1.2\right] \pm 0.1 \mathrm{gw}$

Fig. $4 \mathrm{~W}(4$ to 10$)=\left[23.87\left(1000 / d^{2}\right)-1.22\right] \pm 0.07 \mathrm{gw}$

Fig. $5 W(4 t o 11)=\left[24.84\left(1000 / d^{2}\right)-1.11\right] \pm 0.07 \mathrm{gw}$

Fig. $6 W(4 t o 12)=\left[24.52\left(1000 / d^{2}\right)-1.74\right] \pm 0.08 \mathrm{gw}$

Fig. $7 W(4 t o 15)=\left[26.57\left(1000 / d^{2}\right)-1.24\right] \pm 0.09 \mathrm{gw}$

Fig. $8 W(4 t o 20)=\left[28.98\left(1000 / d^{2}\right)-1.22\right] \pm 0.09 \mathrm{gw}$

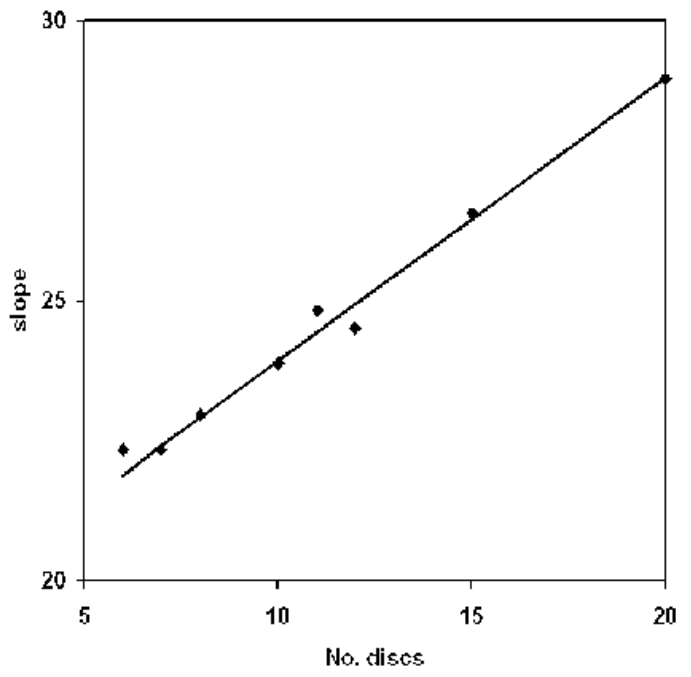

Figure 9: Plot of the slopes of the lines in Figures $1-8$.

The line in Fig. 9 of the SLOPE of the lines in Figures $1-8$ is related to the number $N$ of disks in the upper support by:

$$
S L O P E=(0.51 N+18.9) \pm 0.2
$$




\section{THE EXPERIMENT}

\subsection{EXPERIMENT 2: NEUTRINO}

The following plots the $W$ versus the angle $A$ of rotation for each of the particle configurations.

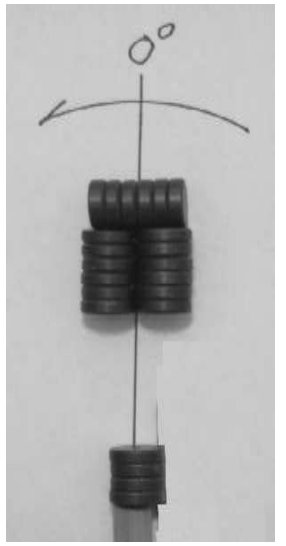

Figure 10: Photograph of the neutrino magnetic configuration from the "flat" side. The arrow indicates the direction of rotation.

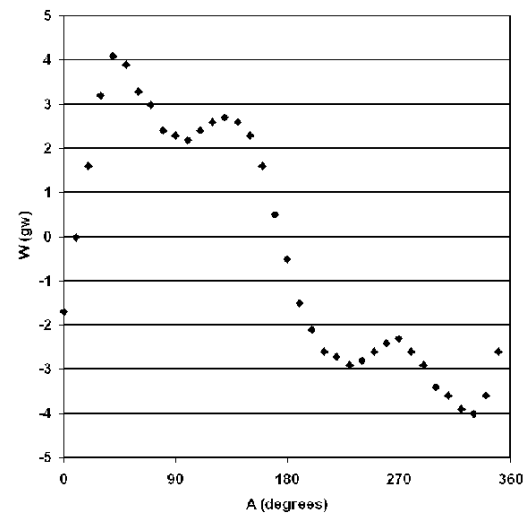

Figure 12: Plot of $W$ versus $A$ for the "flat" rotation.

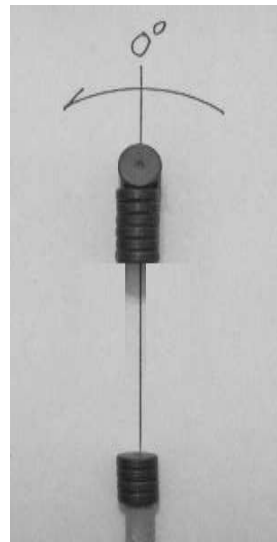

Figure 11: Photograph of the neutrino magnetic configuration from the "side" side. The arrow indicates the direction of rotation.

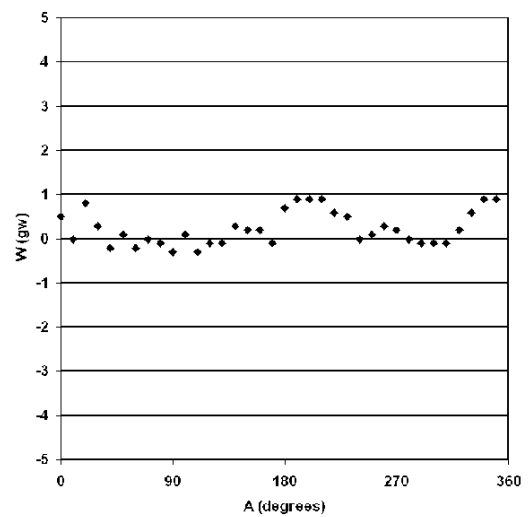

Figure 13: Plot of $W$ versus $A$ for the "side" rotation. 


\subsection{EXPERIMENT 3: ELECTRON}

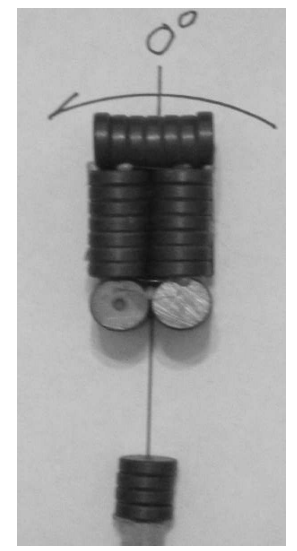

Figure 14: Photograph of the electron magnetic configuration from the "flat" side. The arrow indicates the direction of rotation.

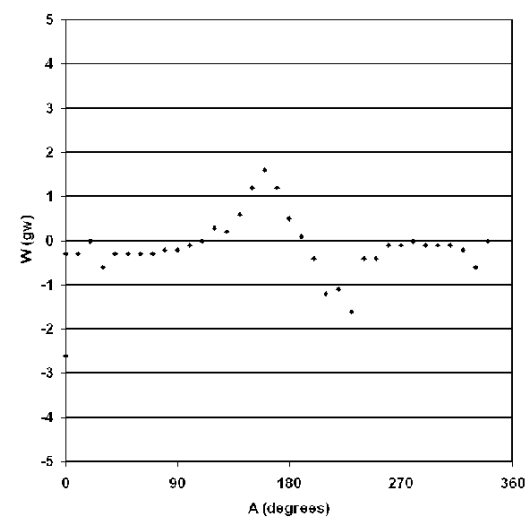

Figure 16: Plot of $W$ versus $A$ for the "flat" rotation.

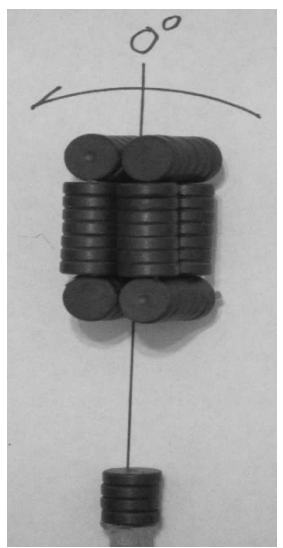

Figure 15: Photograph of the electron magnetic configuration from the "edge" side. The arrow indicates the direction of rotation.

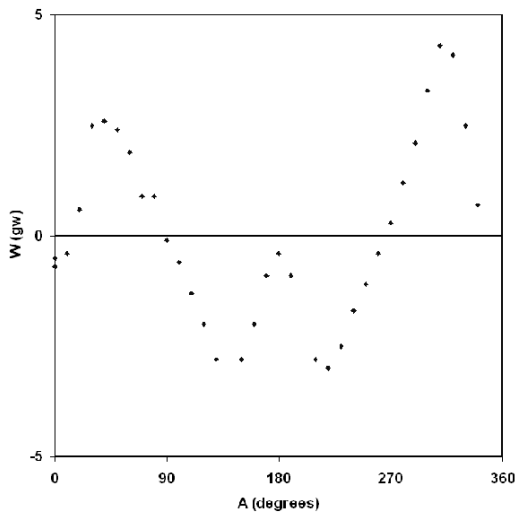

Figure 17: Plot of $W$ versus $A$ for the "edge" rotation. 


\subsection{EXPERIMENT 4: POSITRON}

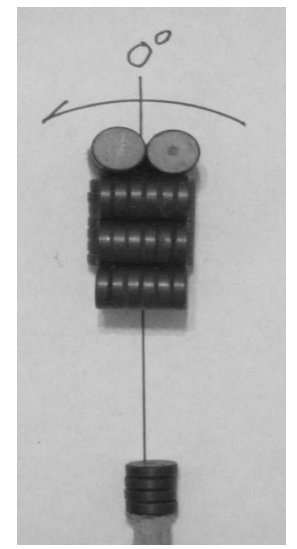

Figure 18: Photograph of the positron magnetic configuration from the "flat" side. The arrow indicates the direction of rotation.

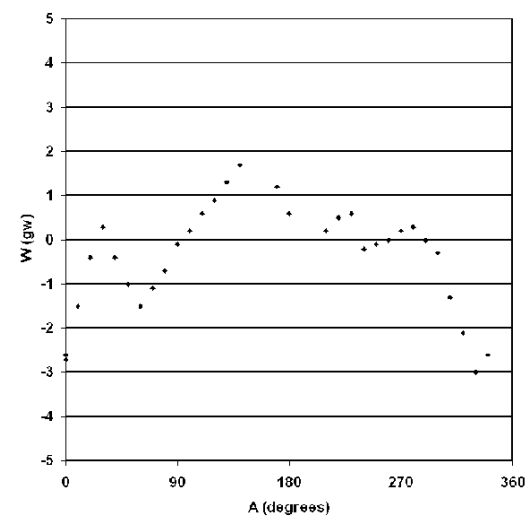

Figure 20: Plot of $W$ versus $A$ for the "flat" rotation.

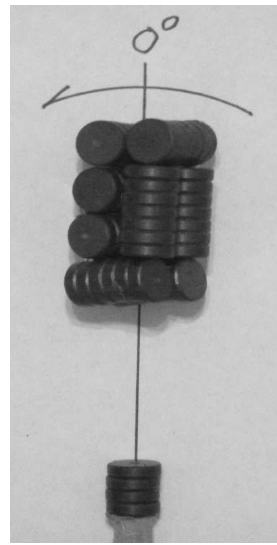

Figure 19: Photograph of the positron magnetic configuration from the "edge" side. The arrow indicates the direction of rotation.

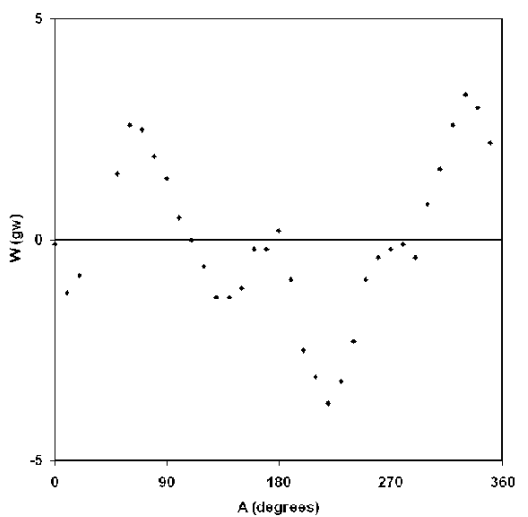

Figure 21: Plot of $W$ versus $A$ for the "edge" rotation. 


\section{DISCUSSION AND CONCLUSION}

The characterization of magnetic fields around elementary particles shows lobes of magnetic field strength around particles separated by directions of little variation between the lobes. The Stern-Gerlach experiment and the fermionic nature of particles follow from the lobe structure of the magnetic field of particles (Hodge 2016b). Because the South Pole was used to measure field strength, the slightly repulsive force measured between lobes is South Pole strength such as seen in Fig. 13. This confirms the idea of the South Pole having slightly stronger force than the North Pole as seen in Hodge (2019). However, this measurement is within the error limits of the setup.

\section{References}

Hodge, J.C., 2016a, Structure and spin of the neutrino, electron, and positron, IntellectualArchive, Vol.5, No. 2, P. 1,. http://intellectualarchive.com/?link=item\&id=1694

Hodge, J.C., 2016b, STOE model of the spin 1/2 observation, IntellectualArchive, Vol.5, No. 4, P. 1 ,. http://intellectualarchive.com/?link=item\&id=1735

Hodge, J.C., 2018a, Magnetostatics relation to gravity with experiment that rejects Biot-Savart Law, IntellectualArchive, Vol.7, No. 3, P. 1,. http://intellectualarchive.com/?link=item\&id=1945

Hodge, J.C., 2018b, Another ecperiment rejects Ampere's Law and supports the STOE model, IntellectualArchive, Vol.7, No. 4, P. 6,. http://intellectualarchive.com/?link=item\&id=1956

Hodge, J.C., 2018c, Two different types of magnetic field, IntellectualArchive, Vol.4, No. 4, P. 1,. http://intellectualarchive.com/?link=item\&id=1964

Hodge, J.C., 2019, Magnetic field evolves to gravity field Part 1: Repulsion , http://intellectualarchive.com/?link=item\&id=2164 